\title{
GEOESTATÍSTICA PARA A AVALIAÇÃO DO CONTROLE AMBIENTAL DO SISTEMA DE VENTILAÇÃO EM INSTALAÇÕES COMERCIAIS PARA FRANGOS DE CORTE
}

\author{
THAYLA M. R. DE C. CURI ${ }^{1}$, RIMENA DO A. VERCELLINO ${ }^{2}$, JULIANA M. MASSARI ${ }^{3}$, \\ ZIGOMAR M. SOUZA ${ }^{4}$, DANIELLA J. DE MOURA ${ }^{5}$
}

\begin{abstract}
RESUMO: O sistema de ventilação e de resfriamento utilizado em instalações para frangos de corte desempenha um papel importante no controle ambiental, possibilitando que a ave expresse seu potencial máximo de produção. Nesse sentido, o objetivo deste trabalho foi avaliar, por meio da geoestatística, a influência do sistema de ventilação e das diferentes tipologias construtivas em relação ao ambiente térmico e aéreo em aviários de frangos de corte na fase final de criação. Os aviários avaliados com sistema de ventilação artificial de pressão negativa, por meio de exaustores, foram: Blue House com nebulizadores na entrada de ar e vedação de cortinas; Dark House com painel evaporativo (tijolo cerâmico) e vedação de cortinas e Solid Wall com painel evaporativo (celulose) e vedação de alvenaria. Os atributos avaliados foram: temperatura, umidade relativa, velocidade do ar, concentração de dióxido de carbono e amônia, durante o período de verão às 14h, em 52 pontos equidistantes, no interior do aviário. Os dados foram avaliados pela análise estatística e geoestatística. A análise geoestatística apresentou-se como ferramenta eficiente para a avaliação da distribuição das condições ambientais nos aviários, e a presença ou não do painel evaporativo influenciou na condição padrão de funcionamento do sistema de ventilação.
\end{abstract}

PALAVRAS-CHAVE: avicultura, conforto térmico, qualidade do ar, sistema de ventilação.

\section{GEOSTATISTIC TO EVALUATE THE ENVIRONMENTAL CONTROL IN DIFFERENT VENTILATION SYSTEMS IN BROILER HOUSES}

\begin{abstract}
The ventilation and cooling systems are important to environmental control inside broilers house enabling the bird to express the maximum potential of production. The aim of this study was to evaluate, using geostatistical analysis, the influence of the ventilation system and the different typologies of building in relation to thermal welfare and air quality in broilers house with birds in the final phase. The broiler facilities evaluated were: Blue House - ventilation systems with exhaust fans and nebulizers, Dark House - ventilation systems with exhaust fans and pad cooling and Solid Wall - ventilation systems with exhaust fans and pad cooling. The attributes evaluated were dry bulb temperature, relative humidity, air velocity, concentration of carbon dioxide and ammonia, during the summer time at 2PM on 52 equidistant points inside the building. The data analysis was by statistical and geostatistical. Geostatistical analysis was an efficient tool to evaluate distribution of environmental conditions in the broilers houses and was able to identify the influence of presence or absence of evaporative panel inside conditions.
\end{abstract}

KEYWORDS: air quality, poultry, thermal welfare, ventilation system.

\section{INTRODUÇÃo}

A avicultura de corte tem buscado o progresso por meio de pesquisas nas áreas de genética, instalações, nutrição, manejo, sanidade e conforto ambiental. Todos esses estudos têm como

\footnotetext{
${ }^{1}$ Enga $^{\text {a }}$ grícola, Dra., Faculdade de Engenharia Agrícola, UNICAMP/Campinas - SP, Fone: (19) 98162-3050,

thaylamrcarvalho@hotmail.com.

${ }^{2}$ Zootecnista, Mestre, Faculdade de Engenharia Agrícola, UNICAMP/Campinas - SP, rimena.vercellino@gmail.com.

${ }^{3}$ Médica Veterinária, Mestre, Faculdade de Engenharia Agrícola, UNICAMP/Campinas - SP, jujumassari@hotmail.com.

${ }^{4}$ Eng ${ }^{\circ}$ Agrícola, Prof. Dr., Faculdade de Engenharia Agrícola, UNICAMP/Campinas - SP, zigomarms@feagri.unicamp.br.

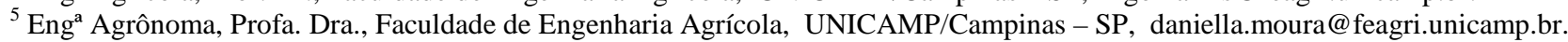

Recebido pelo Conselho Editorial em: 02-7-2014

Aprovado pelo Conselho Editorial em: 24-9-2014
}

Eng. Agríc., Jaboticabal, v.34, n.6, p. 1062-1074, nov./dez. 2014 
propósito compreender melhor os fatores que influenciam o desenvolvimento e o desempenho de frangos de corte (NAZARENO et al., 2009). Dentre os diversos fatores que influenciam na produção de frangos de corte, os fatores ambientais, como a temperatura, umidade relativa, iluminância, radiação, entre outros, assumem relevante importância no processo de criação dos animais por comprometer a função vital mais importante das aves, a homeotermia (AMARAL et al., 2011).

É sabido que os animais atingem sua produtividade ótima quando são mantidos em ambiente termoneutro, ou seja, quando a energia do alimento não é desviada para compensar desvios térmicos em relação ao intervalo de termoneutralidade para eliminar ou manter seu calor. Neste sentido, o ambiente gerado pelo sistema de ventilação nas instalações de frango de corte desempenha papel fundamental no controle de vários fatores ambientais, diluindo o ar interno por meio do ar fresco externo, uma vez que apresenta função sanitária para a retirada do excesso da umidade, da poeira, dos odores e gases, e para prover oxigênio às aves, e função térmica para a retirada do calor produzido pelas aves, equipamentos, ganho de calor pela radiação solar e iluminação (BUCKLIN et al., 2009; MOSTAFA et al., 2012; CORKERY et al., 2013). Neste sentido, aves na fase final de produção estão em conforto térmico quando a temperatura se apresenta entre 15 e $25{ }^{\circ} \mathrm{C}$, e a umidade relativa do ar, entre 50 a 70\% (NICHOLSON et al., 2004; MELUZZI \& SIRRI, 2009). A velocidade do ar é um parâmetro que mais influencia na perda de calor sensível, com valor ideal de $2 \mathrm{~m} \mathrm{~s}^{-1}$ para frangos de corte até a fase final de crescimento (DOZIER et al., 2006). Em relação à qualidade do ar, algumas instituições, como o GLOBALGAP (2012), estabeleceram critérios para o ambiente aéreo de aviários, sendo o limite máximo de 20 ppm para a concentração de amônia e 5.000 ppm para a concentração de dióxido de carbono.

Ambientes considerados quentes e úmidos, mesmo sem haver mortalidade apresentaram redução em $67 \%$ no ganho de peso diário. Por esta razão, é fundamental explorar a eficiência do sistema de ventilação a fim de melhorar todo o projeto de construção e tipologia, melhorando o ambiente interno (BUSTAMANTE et al., 2013). Atualmente, a necessidade do controle efetivo do ambiente interno (térmico e aéreo) nas instalações para criação de frangos de corte é a principal razão do uso de diferentes métodos de simulação para profissionais da área, tais como a geoestatística (BARBOSA FILHO et al., 2009; BORGES et al., 2010; YANAGI JÚNIOR et al., 2011; CARVALHO et al., 2011; CARVALHO et al., 2012; PEREIRA et al., 2012; SILVA et al., 2012; CHOWDHURY et al., 2013; SILVA et al., 2013). A geoestatística é uma ferramenta que permite a interpretação dos resultados baseada na estrutura de sua variabilidade natural, por meio do cálculo da dependência espacial (VIEIRA, 2000). Portanto, o conhecimento da variabilidade espacial dos atributos ambientais e a construção de mapas de krigagem podem auxiliar no manejo correto da ventilação. Assim, o presente trabalho teve como objetivo avaliar e estudar os sistemas de ventilação em relação à qualidade do ambiente térmico e aéreo no interior das instalações comerciais de frangos de corte, por meio da geoestatística.

\section{MATERIAL E MÉTODOS}

O presente trabalho foi conduzido em três aviários comerciais, localizados na região de Amparo-SP, com clima Cwa com duas estações bem definidas (conforme classificação de Köeppen).

O registro dos dados foi realizado semanalmente, quando as aves da linhagem Cobb Vantress 500 apresentavam 28; 35 e 42 dias de idade, às 14h, no período de verão do ano de 2012.

O aviário do tipo Blue House (BH), aviário com sistema de ventilação artificial do tipo túnel de pressão negativa com isolamento lateral por cortinas azuis na face interna e prata na face externa, para o controle da luminosidade, estava localizado em Amparo-SP (latitude $22^{\circ} 45^{\prime} 43^{\prime \prime}$ sul, longitude $46^{\circ} 47^{\prime} 43^{\prime \prime}$ oeste, altitude de $706 \mathrm{~m}$ ), com orientação cartográfica noroeste-sudeste, dimensões de 17 x 90 x 2,45 m (largura x comprimento x pé-direito) e densidade de 15 aves $\mathrm{m}^{-2}$. O sistema de ventilação era artificial de pressão negativa, contendo dez exaustores, com diâmetro de 
1,38 m, hélice com três pás e motor de 735,5 W e vazão nominal de 10,56 $\mathrm{m}^{3} \mathrm{~s}^{-1}$ (à pressão de zero Pascal), sem uso de painel evaporativo e vedação com cortinas laterais na cor azul na face interna e prata na face externa.

O aviário do tipo Dark House (DH), aviário com sistema de ventilação artificial do tipo túnel de pressão negativa com isolamento lateral por cortinas pretas na face interna e prata na face externa, para o controle da luminosidade, estava localizado em Amparo-SP (latitude $22^{\circ} 44^{\prime} 01^{\prime \prime}$ sul, longitude 46 44'02" oeste, altitude de $706 \mathrm{~m}$ ), com orientação cartográfica noroeste-sudeste, dimensões de 20 x 150 x 2,90 m (largura x comprimento x pé-direito) e densidade de 12 aves m$^{-2}$. O sistema de ventilação era artificial de pressão negativa, contendo dezesseis exaustores, com diâmetro de 1,30 m, hélice com três pás e motor de 1.103,25 W e vazão nominal de $10,56 \mathrm{~m}^{3} \mathrm{~s}^{-1}$ (à pressão de zero Pascal), com uso de painel evaporativo feito de tijolo cerâmico e vedação com cortinas laterais na cor preta na face interna e prata na face externa.

O aviário do tipo Solid Wall (SW), aviário com sistema de ventilação artificial do tipo túnel de pressão negativa com isolamento lateral por paredes de alvenaria para controle da luminosidade, estava localizado em Amparo-SP (latitude 22 45'37" sul, longitude 4646'42" oeste, altitude de 706 m), com orientação cartográfica norte-sul, dimensões de 20 x 120 x 3 m (largura x comprimento x pé-direito) e densidade de 13 aves $\mathrm{m}^{-2}$. O sistema de ventilação era artificial de pressão negativa, contendo dezesseis exaustores, com diâmetro de 1,38 m, hélice com três pás e motor de 735,5 W e vazão nominal de 11,42 $\mathrm{m}^{3} \mathrm{~s}^{-1}$ (à pressão de zero Pascal), com uso de painel evaporativo feito de celulose e vedação de alvenaria com parede de blocos de concreto.

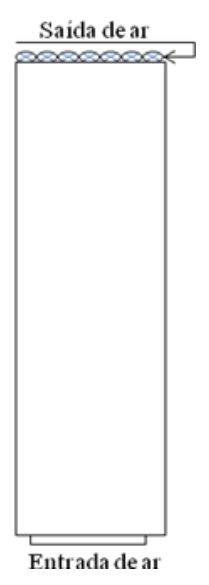

(a)

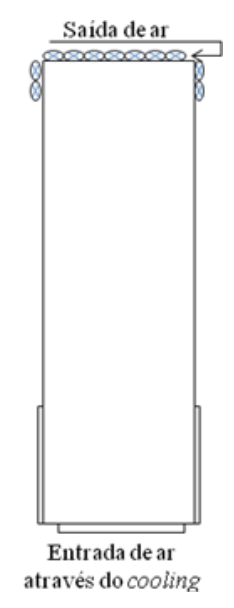

(b)

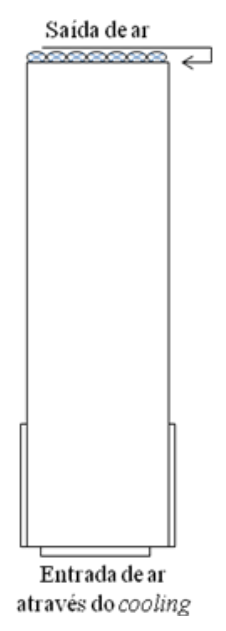

(c)

FIGURA 1. Esquema simplificado dos aviários estudados do tipo Blue House (a), Dark House (b) e Solid Wall (c). Scheme of broiler houses such as Blue House (a), Dark House (b) and Solid Wall (c).

O registro dos dados de temperatura $\left(\mathrm{Tbs},{ }^{\circ} \mathrm{C}\right)$ e velocidade do ar $\left(\mathrm{Var}, \mathrm{m} \mathrm{s}^{-1}\right)$ foi realizado por meio de um anemômetro de fio quente (VelociCalc ${ }^{\circledR}, \mathrm{TSI}_{\mathrm{TM}}$ ), com escala de leitura para temperatura de -18 a $93{ }^{\circ} \mathrm{C}$ e resolução $0,1{ }^{\circ} \mathrm{C}$, e velocidade do ar com amplitude de 0 a $30 \mathrm{~m} \mathrm{~s}^{-1}$, resolução de velocidade de $0,01 \mathrm{~m} \mathrm{~s}^{-1}$ e exatidão de $\pm 0,015 \mathrm{~m} \mathrm{~s}^{-1}$. Para os dados de umidade relativa (UR, \%), foram coletados por meio do equipamento THDL 400 (Instrument ${ }^{\circledR}$ ), com amplitude de 25 a $95 \%$ e precisão de $\pm 5 \%$. Os dados de concentração de amônia $\left(\mathrm{NH}_{3}\right.$, $\left.\mathrm{ppm}\right)$ e concentração de dióxido de carbono $\left(\mathrm{CO}_{2}\right.$, $\left.\mathrm{ppm}\right)$ foram registrados por meio de um medidor instantâneo de concentração de gases (GasAlertMIcro 5, BW Technologies ${ }^{\circledR}$ ).

Nos dados de temperatura de bulbo seco e umidade relativa, foram realizadas correções numéricas adaptadas da metodologia proposta por BARBOSA FILHO et al. (2009) que consiste em 
corrigir a defasagem de tempo entre a coleta do dado no primeiro e no último ponto, em decorrência da coleta manual dos dados.

A variabilidade dos atributos foi verificada baseada na estatística descritiva dos dados registrados (Tbs, UR, Var, $\mathrm{CO}_{2}$ e $\mathrm{NH}_{3}$ ), obtendo-se valores da média, mediana, coeficiente de variação e o teste de normalidade de Kolmogorov-Smirnov. A análise estatística foi realizada, utilizando o programa computacional estatístico SAS ${ }^{\circledR-}$.

A dependência espacial foi analisada por meio de ajustes de semivariogramas (VIEIRA, 2000), com base na pressuposição de estacionariedade da hipótese intrínseca, a qual é estimada pela [eq. (1)].

$$
\hat{\gamma}=\frac{1}{2 N(h)} \sum_{i=1}^{N(h)}\left[Z\left(x_{1}\right)-Z\left(x_{1}+h\right)\right]^{2}
$$

em que,

$\mathrm{N}(\mathrm{h})$ - número de pares experimentais;

$\mathrm{Z}\left(\mathrm{x}_{1}\right)$ e $\mathrm{Z}\left(\mathrm{x}_{1}+\mathrm{h}\right)$ - observações separadas por uma distância $\mathrm{h}(\mathrm{m})$.

O semivariograma é representado no gráfico pela semivariância versus h. Do ajuste de um modelo matemático aos valores calculados da semivariância, são estimados os coeficientes do modelo teórico para o semivariograma (o efeito pepita $-\mathrm{C}_{0}$, m; patamar, $\mathrm{C}_{0}+\mathrm{C}_{1}$, m; e o alcance, a, m). Para analisar o grau da dependência espacial dos atributos em estudo, utilizou-se da classificação de CAMBARDELLA et al. (1994), em que são considerados como grau da dependência espacial forte os semivariogramas que têm um efeito pepita $<25 \%$ do patamar, moderada quando está entre 25 e $75 \%$ e fraca $>75 \%$.

Na determinação da existência ou não da dependência espacial, utilizou-se do exame de semivariogramas, através do programa GS+. A elaboração dos mapas de distribuição espacial das variáveis foi realizada no programa SURFER v.9. Com o objetivo de facilitar a comparação do grau de dependência espacial das variáveis em estudo, o efeito pepita foi expresso como percentagem do patamar (TRANGMAR et al., 1985).

\section{RESULTADOS E DISCUSSÃO}

De acordo com o teste de Kolmogorov-Smirnov, as variáveis de Tbs e UR para o aviário SW, Var para os aviários $\mathrm{BH}$ e $\mathrm{DH}$, e concentração de $\mathrm{NH}_{3}$ para os aviários $\mathrm{BH}$ e $\mathrm{SW}$, aos 28 dias de idade das aves, UR para o aviário $\mathrm{DH}$, Var para os aviários $\mathrm{BH}$ e $\mathrm{SW}$, e a concentração de $\mathrm{NH}_{3}$ para o aviário SW, aos 35 dias de idade, e Tbs e Var para o DH, UR para o DH e SW, Var para o $\mathrm{BH}$ e $\mathrm{DH}$, e para a concentração de $\mathrm{NH}_{3}$ para os três aviários avaliados, aos 42 dias de idade das aves, apresentaram normalidade (Tabela 1). Com exceção da concentração de $\mathrm{CO}_{2}$, as variáveis avaliadas apresentaram valores próximos de média e mediana, o que permite assumir que as distribuições são suficientemente simétricas. Segundo LITTLE \& HILLS (1978), quando o valor da média e da mediana estão próximos, os dados aproximam-se da distribuição normal. Isso pode ser um indicativo de que as medidas de tendência central não são dominadas por valores atípicos na distribuição (CAMBARDELLA et al., 1994).

Em relação à variabilidade dos dados ambientais classificada em baixa (coeficiente de variação abaixo de 12\%), média (coeficiente de variação entre 12 e 24\%) e alta (coeficiente de variação acima de 24\%), de acordo com a literatura (WARRICK \& NIELSEN, 1980), foi possível observar que, aos 28 dias de idade das aves, a Tbs para os três aviários avaliados e para a UR no SW, aos 35 e aos 42 dias de idade, as variáveis de Tbs e UR para os três aviários avaliados, ocorreu baixa variabilidade dos dados. A média variabilidade dos dados foi observada para a UR nos aviário $\mathrm{BH}$ e $\mathrm{DH}$ aos 28 dias de idade e para a concentração de $\mathrm{NH}_{3}$ no aviário $\mathrm{DH}$ aos 42 dias de idade das aves. A Var, concentração de $\mathrm{CO}_{2}$ e $\mathrm{NH}_{3}$ para os aviários $\mathrm{BH}, \mathrm{DH}$ e $\mathrm{SW}$ apresentaram alta 
variabilidade aos 28 e 42 dias de idade das aves, da mesma forma que a Var e concentração de $\mathrm{CO}_{2}$ para os três aviários e concentração de $\mathrm{NH}_{3}$ para o $\mathrm{BH}$ e $\mathrm{SW}$ aos 35 dias de idade das aves. A avaliação da homogeneidade dos parâmetros climáticos é essencial para assegurar a qualidade do manejo do ambiente térmico e aéreo, a fim de minimizar a ocorrência de regiões quentes e com pouca renovação de ar (pontos mortos), melhorando a qualidade e a produção do lote (BOURNET \& BOULARD, 2010).

TABELA 1. Resultado da análise estatística das variáveis ambientais para os diferentes aviários (BH, DH e SW), aos 28; 35 e 42 dias de vida das aves, no período do verão e no horário das $14 \mathrm{~h}$. Descriptive statistics for environmental data collected from different broiler houses (BH, DH and SW), when the birds were 28, 35 and 42 days old, on summer and at 2PM.

\begin{tabular}{|c|c|c|c|c|c|c|c|c|c|c|c|c|c|}
\hline & & \multicolumn{4}{|c|}{28 dias de idade } & \multicolumn{4}{|c|}{35 dias de idade } & \multicolumn{4}{|c|}{42 dias de idade } \\
\hline Atr & Trat & $\mathrm{M}$ & Med & $\mathrm{CV}$ & $\mathrm{d}$ & $\mathrm{M}$ & Med & $\mathrm{CV}$ & $\mathrm{d}$ & $\mathrm{M}$ & Med & $\mathrm{CV}$ & $\mathrm{d}$ \\
\hline \multirow{3}{*}{$\begin{array}{l}\text { Tbs } \\
{\left[{ }^{\circ} \mathrm{C}\right]}\end{array}$} & $\mathrm{BH}$ & 28,76 & 28,82 & 2,19 & 0,04 & 29,00 & 28,46 & 5,84 & 0,01 & 29,98 & 30,03 & 1,32 & 0,04 \\
\hline & $\mathrm{DH}$ & 26,73 & 26,46 & 3,99 & 0,01 & 24,35 & 24,28 & 3,52 & 0,04 & 24,25 & 24,29 & 2,91 & $0,07^{\mathrm{ns}}$ \\
\hline & SW & 25,45 & 25,64 & 4,24 & $0,15^{\mathrm{ns}}$ & 27,39 & 27,27 & 3,74 & 0,04 & 25,35 & 24,98 & 4,31 & 0,01 \\
\hline \multirow{3}{*}{$\begin{array}{c}\text { UR } \\
\text { [\%] }\end{array}$} & $\mathrm{BH}$ & 49,39 & 52,60 & 19,07 & 0,01 & 52,15 & 52,85 & 7,18 & 1 & 45,12 & 44,69 & 10,59 & 0,01 \\
\hline & $\mathrm{DH}$ & 81,17 & 82,63 & 12,58 & 0,01 & 77,46 & 78,16 & 10,31 & $0,14^{\mathrm{ns}}$ & 81,24 & 80,70 & 5,92 & $0,15^{\mathrm{ns}}$ \\
\hline & SW & 78,51 & 79,08 & 6,71 & $0,07^{\mathrm{ns}}$ & 80,97 & 79,85 & 4,07 & 1 & 90,04 & 89,96 & 2,32 & $0,15^{\mathrm{ns}}$ \\
\hline \multirow{3}{*}{$\begin{array}{c}\mathrm{Var} \\
{\left[\mathrm{m} \mathrm{s}^{-1}\right]}\end{array}$} & $\mathrm{BH}$ & 1,58 & 1,54 & 26,28 & $0,15^{\mathrm{ns}}$ & 1,15 & 1,09 & 30,09 & $0,15^{\mathrm{ns}}$ & 1,32 & 1,34 & 32,63 & $0,15^{\mathrm{ns}}$ \\
\hline & $\mathrm{DH}$ & 1,21 & 1,22 & 25,53 & $0,15^{\mathrm{ns}}$ & 1,29 & 1,20 & 27,76 & 0,01 & 1,24 & 1,21 & 30,28 & $0,05^{\mathrm{ns}}$ \\
\hline & SW & 0,36 & 0,33 & 34,03 & 0,04 & 0,91 & 0,87 & 29,15 & $0,15^{\mathrm{ns}}$ & 1,23 & 1,15 & 26,50 & 0,01 \\
\hline \multirow{3}{*}{$\begin{array}{c}\mathrm{CO}_{2} \\
{[\mathrm{ppm}]}\end{array}$} & $\mathrm{BH}$ & 6,38 & 0,00 & 368,73 & 0,03 & 6,33 & 0,00 & 371.32 & 0,01 & 0,00 & 0,00 & - & - \\
\hline & $\mathrm{DH}$ & 6,25 & 0,00 & 376,79 & 0,01 & 62,74 & 18,75 & 108,30 & 0,01 & 36,78 & 0,00 & 155,74 & 0,01 \\
\hline & SW & 284,90 & 312,50 & 61,23 & 0,01 & 28,12 & 0,00 & 248,92 & 0,01 & 23,80 & 0,00 & 225,08 & 0,01 \\
\hline \multirow{3}{*}{$\begin{array}{c}\mathrm{NH}_{3} \\
{[\mathrm{ppm}]}\end{array}$} & $\mathrm{BH}$ & 4,60 & 4,25 & 42,04 & $0,05^{\mathrm{ns}}$ & 2,45 & 3,00 & 64,77 & 0,01 & 2,82 & 3,00 & 50,90 & $0,15^{\mathrm{ns}}$ \\
\hline & $\mathrm{DH}$ & 2,89 & 3,00 & 46,46 & 0,01 & 6,73 & 7,00 & 18,16 & 0,01 & 5,03 & 5,00 & 33,32 & $0,15^{\mathrm{ns}}$ \\
\hline & SW & 11,35 & 11,88 & 43,87 & $0,15^{\mathrm{ns}}$ & 4,05 & 4,00 & 31,63 & $0,07^{\mathrm{ns}}$ & 4,73 & 4,50 & 57,69 & $0,09^{\mathrm{ns}}$ \\
\hline
\end{tabular}

Atri = Variáveis; Trat = Aviários; $\mathrm{M}=$ média; Med = mediana; $\mathrm{CV}=$ Coeficiente de Variação; $\mathrm{d}=$ teste de normalidade por Kolmogorov-Smirnov; Tbs = temperatura de bulbo seco; UR = umidade relativa; Var = velocidade do ar; $\mathrm{CO}_{2}=$ concentração de dióxido de carbono; $\mathrm{NH}_{3}=$ concentração de amônia; $\mathrm{BH}=$ Blue House; $\mathrm{DH}=$ Dark House; $\mathrm{SW}=$ Solid Wall .

Aos 28 dias de idade das aves, os semivariogramas da Tbs e da concentração de $\mathrm{NH}_{3}$ no aviário BH e da UR no BH e SW foram ajustados no modelo gaussiano, o modelo esférico ajustou-se para a Tbs nos aviários DH e SW, para a Var nos três aviários avaliados, concentração de $\mathrm{CO}_{2}$ e $\mathrm{NH}_{3}$ para SW e DH, respectivamente. A UR para $\mathrm{DH}$, a concentração de $\mathrm{CO}_{2}$ para os aviários $\mathrm{BH}$ e $\mathrm{DH}$ e a concentração de $\mathrm{NH}_{3}$ para o $\mathrm{SW}$ tiveram seus semivariogramas ajustados no modelo exponencial. A Tbs e a UR para os aviários $\mathrm{BH}$ e $\mathrm{DH}$, e a concentração $\mathrm{NH}_{3}$ para o $\mathrm{BH}$ foram ajustadas no modelo gaussiano aos 35 dias de idade das aves, assim como os semivariogramas para a Tbs e a UR em SW,Var para $\mathrm{BH}$ e $\mathrm{SW}$ e concentração de $\mathrm{NH}_{3}$ para $\mathrm{DH}$ foram ajustados no modelo esférico, e no modelo exponencial para a Var em $\mathrm{DH}$, concentração de $\mathrm{CO}_{2}$ e $\mathrm{NH}_{3}$ para os três aviários e para SW, respectivamente. Os semivariogramas para aves com 42 dias de idades foram ajustados em gaussiano para a Tbs em SW, UR para $\mathrm{BH}, \mathrm{DH}$ e $\mathrm{SW}$, concentração de $\mathrm{CO}_{2}$ no SW e concentração de $\mathrm{NH}_{3}$ no SW, esférico para Tbs no DH e Var para os três aviários, e exponencial para a Tbs em Bh e para a concentração de $\mathrm{CO}_{2}$ e $\mathrm{NH}_{3}$ para o aviário $\mathrm{DH}$.

A análise da relação $C_{0} /\left(C_{0}+C_{1}\right)$ mostrou que as variáveis apresentaram grau de dependência espacial forte e/ou moderada (Tabela 2). Quanto maior a dependência espacial, menor será a contribuição do efeito pepita na variabilidade dos dados, consequentemente melhores serão as estimativas na krigagem. Portanto, as distribuições dos atributos microclimáticos e aéreos no espaço não são aleatórias, uma vez que todos apresentaram valores moderados ou fortes para o grau de dependência espacial (CARVALHO et al., 2012).

O alcance representa a distância limite de amostragem em que os pontos coletados estão correlacionados, contribuindo para o planejamento amostral e a definição dos locais onde as 
variáveis climáticas serão amostradas (McBRATNEY \& WEBSTER, 1983). Os dados apresentaram valores de alcance variando de 12 a $90 \mathrm{~m}$.

TABELA 2. Modelos e parâmetros estimados dos semivariogramas experimentais para variáveis ambientais, nos diferentes aviários (BH, DH e SW), aos 28; 35 e 42 dias de vida das aves, no período do verão e no horário das $14 \mathrm{~h}$. Estimated models and parameters of semivariograms in different broiler houses (BH, DH and SW), when the birds were 28, 35 and 42 days old, on summer and at 2 PM.

\begin{tabular}{|c|c|c|c|c|c|c|c|c|c|c|c|c|c|c|c|c|}
\hline \multirow[b]{2}{*}{ Atr } & \multirow[b]{2}{*}{ Trat } & \multicolumn{5}{|c|}{28 dias de idade } & \multicolumn{5}{|c|}{35 dias de idade } & \multicolumn{5}{|c|}{42 dias de idade } \\
\hline & & Mod & $\mathrm{A}$ & GDE & $\mathrm{R}^{2}$ & SQR & Mod & $\mathrm{A}$ & GDE & $\mathrm{R}^{2}$ & SQR & Mod & $\mathrm{A}$ & GDE & $\mathrm{R}^{2}$ & SQR \\
\hline \multirow{3}{*}{$\begin{array}{l}\text { Tbs } \\
{\left[{ }^{\circ} \mathrm{C}\right]}\end{array}$} & $\mathrm{BH}$ & GAU & 14 & 0,24 & 0,94 & $4,26 \mathrm{E}-03$ & GAU & 12 & 0,03 & 0,90 & 3,55E-01 & EXP & 62 & 23,55 & 0,96 & $2,41 \mathrm{E}-04$ \\
\hline & $\mathrm{DH}$ & ESF & 18 & 8,41 & 0,87 & 1,58E-02 & GAU & 20 & 9,64 & 0,84 & 2,00E-02 & ESF & 17 & 0,40 & 0,86 & 2,67E-03 \\
\hline & SW & ESF & 15 & 5,08 & 0,57 & 3,33E-02 & ESF & 17 & 0,09 & 0,93 & $1,52 \mathrm{E}-02$ & GAU & 15 & 0,08 & 0,92 & 3,43E-02 \\
\hline \multirow{3}{*}{$\begin{array}{l}\text { UR } \\
\text { [\%] }\end{array}$} & $\mathrm{BH}$ & GAU & 14 & 0,11 & 0,84 & $8,35 \mathrm{E}+02$ & GAU & 13 & 0,08 & 0,82 & $8,45 \mathrm{E}+00$ & GAU & 15 & 0,04 & 0,85 & $6,04 \mathrm{E}+01$ \\
\hline & $\mathrm{DH}$ & EXP & 46 & 0,07 & 0,92 & $3,68 \mathrm{E}+02$ & GAU & 20 & 14,55 & 0,77 & $2,51 E+02$ & GAU & 24 & 28,16 & 0,84 & $1,52 \mathrm{E}+01$ \\
\hline & SW & GAU & 78 & 16,00 & 0,96 & $9,56 \mathrm{E}+00$ & ESF & 15 & 0,47 & 0,66 & $5,40 \mathrm{E}+00$ & GAU & 16 & 0,29 & 0,88 & 3,61E-01 \\
\hline \multirow{3}{*}{$\begin{array}{c}\mathrm{Var} \\
{\left[\mathrm{m} \mathrm{s}^{-1}\right]}\end{array}$} & $\mathrm{BH}$ & ESF & 14 & 3,47 & 0,97 & 1,19E-04 & ESF & 20 & 25,78 & 0,85 & $3,67 \mathrm{E}-04$ & ESF & 22 & 6,41 & 0,91 & $1,41 \mathrm{E}-03$ \\
\hline & $\mathrm{DH}$ & ESF & 16 & 4,41 & 0,77 & 1,17E-04 & EXP & 25 & 0,26 & 0,95 & 6,01E-05 & ESF & 15 & 0,07 & 0,94 & 4,76E-05 \\
\hline & SW & ESF & 17 & 0,07 & 0,90 & 3,55E-06 & ESF & 15 & 0,16 & 0,88 & 4,58E-05 & ESF & 14 & 0,11 & 0,82 & $1,23 \mathrm{E}-04$ \\
\hline \multirow{3}{*}{$\begin{array}{c}\mathrm{CO}_{2} \\
\text { [ppm] }\end{array}$} & $\mathrm{BH}$ & EXP & 40 & 37,96 & 0,65 & $4,31 \mathrm{E}+04$ & EXP & 57 & 4,93 & 0,89 & $2,21 E+04$ & \multicolumn{5}{|c|}{ Valores iguais à zero } \\
\hline & $\mathrm{DH}$ & EXP & 39 & 0,16 & 0,97 & $1,71 \mathrm{E}+03$ & EXP & 42 & 7,48 & 0,90 & $3,30 \mathrm{E}+05$ & EXP & 50 & 4,37 & 0,95 & $1,24 \mathrm{E}+05$ \\
\hline & SW & ESF & 55 & 4,85 & 0,99 & $7,97 \mathrm{E}+05$ & EXP & 36 & 0,23 & 0,92 & $3,27 E+05$ & GAU & 19 & 0,31 & 0,95 & $1,76 \mathrm{E}+05$ \\
\hline \multirow{3}{*}{$\begin{array}{c}\mathrm{NH}_{3} \\
\text { [ppm] }\end{array}$} & $\mathrm{BH}$ & GAU & 68 & 8,04 & 0,98 & $3,56 \mathrm{E}-01$ & GAU & 67 & 11,90 & 0,97 & $1,12 \mathrm{E}-01$ & GAU & 68 & 5,34 & 0,98 & $1,29 \mathrm{E}-01$ \\
\hline & $\mathrm{DH}$ & ESF & 18 & 0,13 & 0,71 & 8,17E-02 & ESF & 19 & 3,37 & 0,97 & 3,45E-03 & EXP & 89 & 36,18 & 0,87 & 5,82E-02 \\
\hline & SW & EXP & 33 & 13,41 & 0,82 & $2,91 E+01$ & EXP & 45 & 6,31 & 0,86 & $1,49 \mathrm{E}-01$ & GAU & 16 & 0,12 & 0,84 & $2,97 \mathrm{E}+00$ \\
\hline
\end{tabular}

Atri = Variáveis; Trat = Aviários; Mod = Modelo; A = alcance, m; GDE = Grau de Dependência Espacial; $\mathrm{R}^{2}=$ coeficiente de determinação; SQR = Soma dos Quadrados dos Resíduos; Tbs = temperatura de bulbo seco; UR = umidade relativa; Var = velocidade do ar; $\mathrm{CO}_{2}=$ concentração de dióxido de carbono; $\mathrm{NH}_{3}=$ concentração de amônia,; $\mathrm{BH}=$ Blue House; $\mathrm{DH}=$ Dark House; $\mathrm{SW}=$ Solid Wall.

O valor médio da temperatura esteve além do recomendado para os aviários estudados, ou seja, acima do intervalo entre 19,1 e $20,2^{\circ} \mathrm{C}$, capaz de melhorar as características de desempenho zootécnico (ARAUJO et al., 2011). O aviário DH apresentou os menores valores de Tbs, indicando que o sistema de resfriamento foi mais eficaz comparado com os outros aviários (FIGURA 2). O aviário SW, com 28 dias de idade, apresentou áreas com temperaturas maiores $\left(26{ }^{\circ} \mathrm{C}\right)$ e áreas com temperaturas menores $\left(24^{\circ} \mathrm{C}\right)$, remetendo a necessidade de maior controle do sistema de ventilação para manter o ambiente mais homogêneo. 


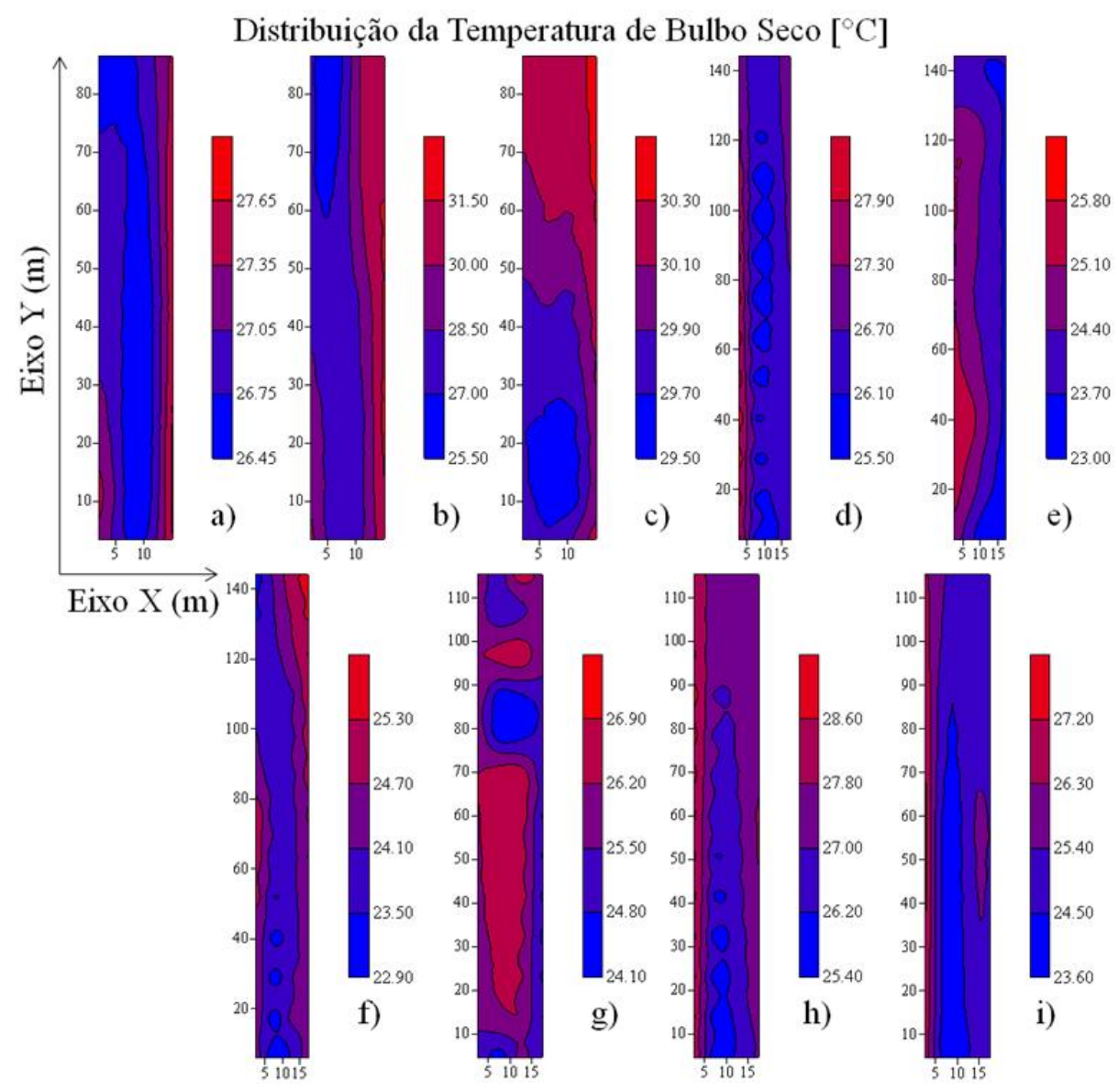

FIGURA 2. Mapas de krigagem para a temperatura de bulbo seco no aviário BH, aos 28 (a), 35(b) e 42(c) dias de idade das aves, DH aos 28 (d), 35(e) e 42(f) dias de idade das aves, e SW aos 28 (g), 35(h) e 42(i) dias de idade das aves, no período do verão, às 14h. Kriging maps for temperature in BH in 28 (a), 35 (b) and 42 (c) days old of birds, DH in 28 (d), 35 (e) and 42 (f) days old of birds and SW in 28 (g), 35 (h) and 42 (i) days old of birds on summer and at 2 PM.

O aviário BH apresentou os maiores valores de Tbs com maior potencial de perda de calor por meios evaporativos, pelos valores de UR se apresentarem próximos de $50 \%$, sendo que a literatura recomenda entre 50 e 70\% (COBB, 2013). Foi possível observar ilhas do centro dos aviários com valores de Tbs mais amenas em função do sistema de resfriamento por painel evaporativo no DH e SW (SILVA et al., 2013), e os maiores valores estavam próximos às entradas de ar através do painel evaporativo (FIGURA 3). Sendo que os aviários DH e SW apresentaram valores de UR acima do recomendado em função do sistema de ventilação tipo túnel, que tende a apresentar maiores níveis de UR em relação ao recomendado (CARVALHO et al., 2012). A Tbs alta, associada à UR do ar alta, dificulta as perdas de calor do animal (ROCHA et al., 2010). 


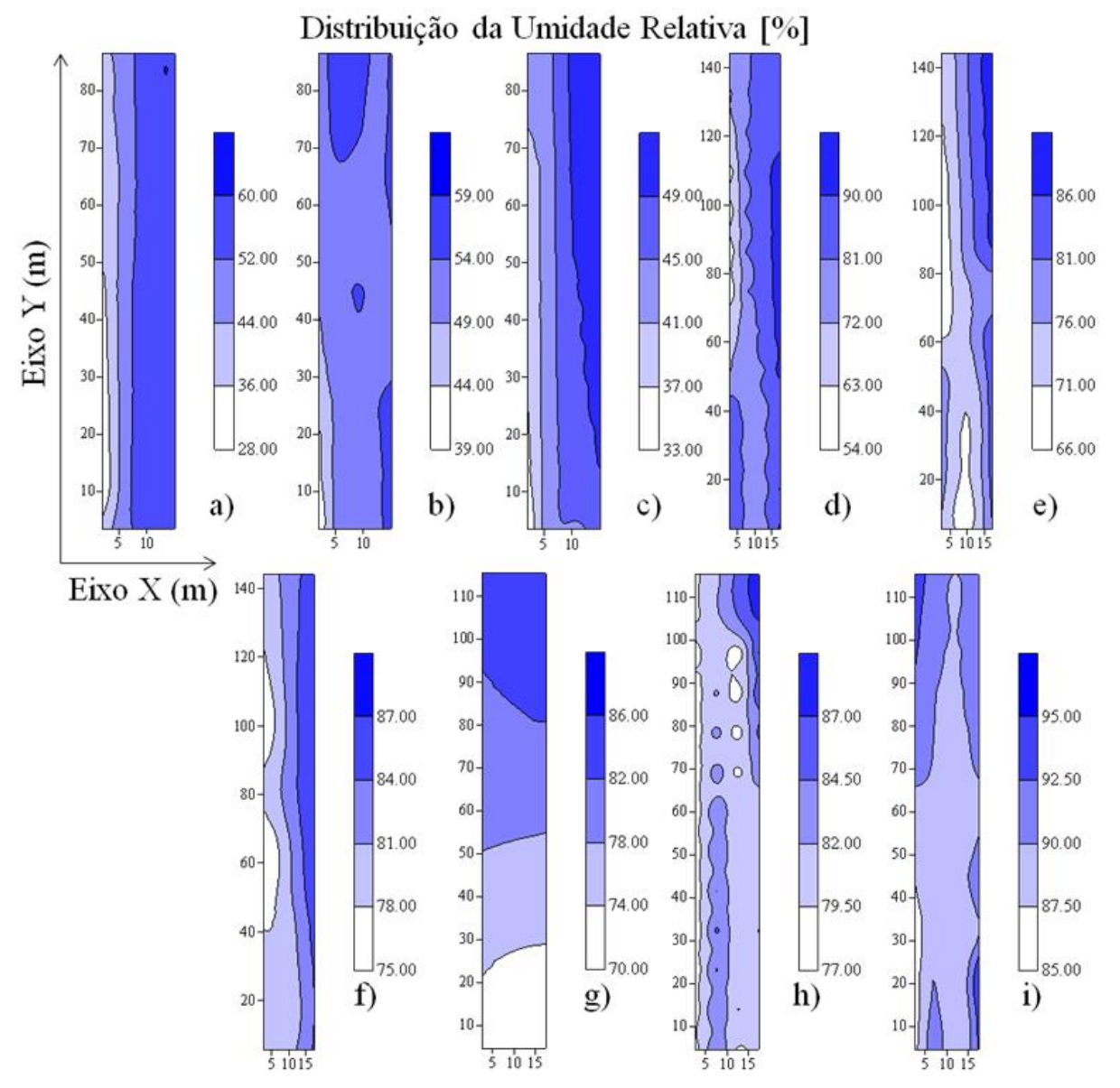

FIGURA 3. Mapas de krigagem para a umidade relativa no aviário BH, aos 28 (a), 35(b) e 42(c) dias de idade das aves, DH aos 28 (d), 35(e) e 42(f) dias de idade das aves, e SW aos 28 (g), 35(h) e 42(i) dias de idade das aves, no período do verão, às 14h. Kriging maps for relative humidity in BH in 28 (a), 35 (b) and 42 (c) days old of birds, DH in 28 (d), 35 (e) and 42 (f) days old of birds and SW in 28 (g), 35 (h) and 42 (i) days old of birds on summer and at 2 PM.

Os valores registrados da Var nos aviários BH e DH apresentaram-se no intervalo considerado ideal, ou seja, próximo de 1,5 $\mathrm{m} \mathrm{s}^{-1}$ (MEDEIROS et al., 2005), e os menores valores foram observados no aviário SW, indicando necessidade de intensificação do sistema de ventilação. Os maiores valores da velocidade do ar foram observados próximo às entradas de ar, aos 28 dias de idade das aves no aviário BH e aos 42 dias de idade das aves nos aviário DH e SW (FIGURA 4). 


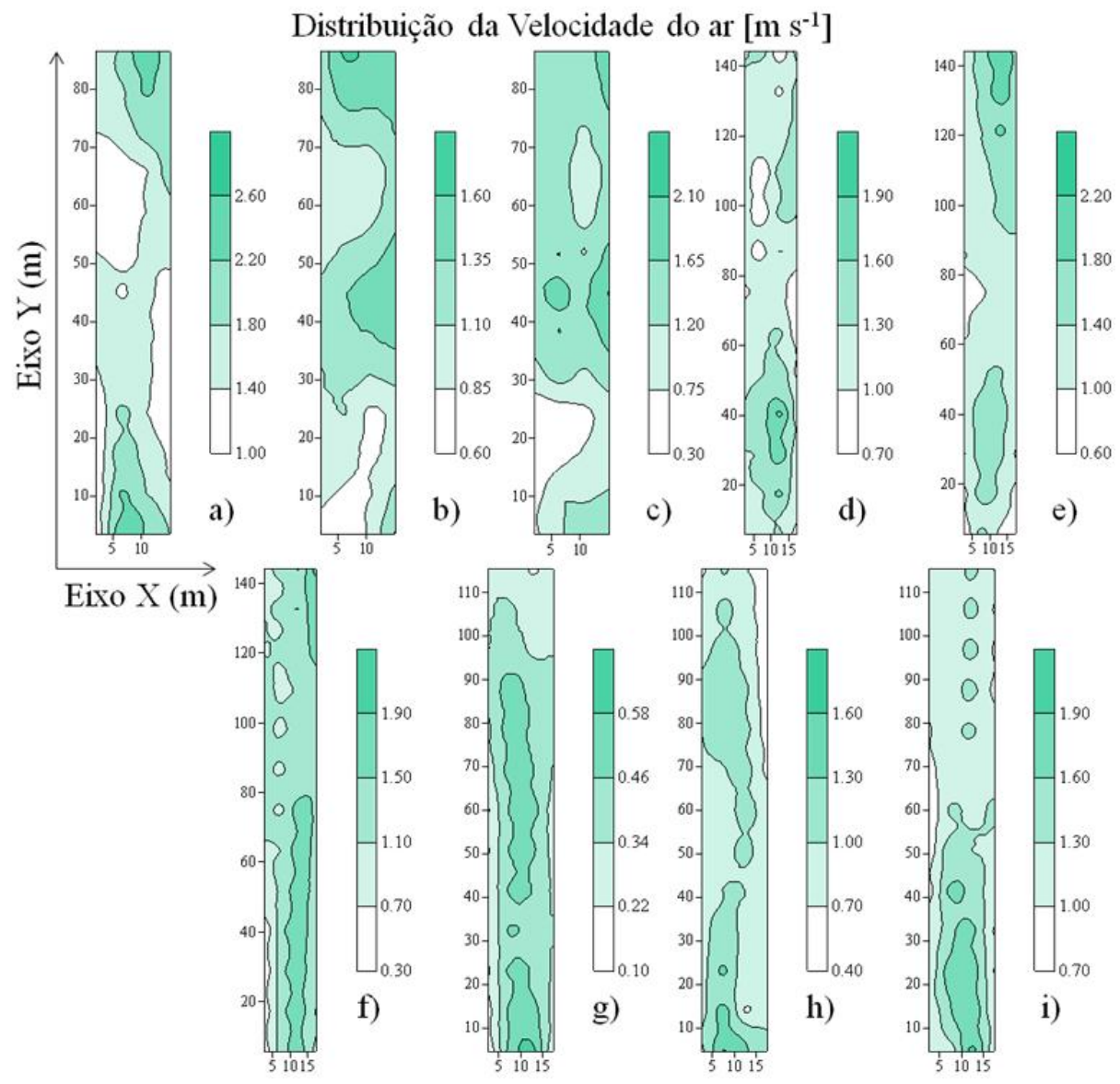

FIGURA 4. Mapas de krigagem para a velocidade do ar no aviário BH, aos 28 (a), 35(b) e 42(c) dias de idade das aves, DH aos 28 (d), 35(e) e 42(f) dias de idade das aves, e SW aos 28 (g), 35(h) e 42(i) dias de idade das aves, no período do verão às $14 \mathrm{~h} 00 \mathrm{~min}$. Kriging maps for air velocity in BH in 28 (a), 35 (b) and 42 (c) days old of birds, DH in 28 (d), 35 (e) and 42 (f) days old of birds and SW in 28 (g), 35 (h) and 42 (i) days old of birds on summer and at 2 PM.

A concentração de $\mathrm{CO}_{2}$ esteve abaixo do limite máximo considerado ideal para todos os aviários, abaixo de 3.000 ppm (GLOBALGAP, 2012), indicando que o sistema de ventilação utilizado pelos aviários estudados foi efetivo para a renovação do ar. Ainda é possível observar maiores valores de $\mathrm{CO}_{2}$ e $\mathrm{NH}_{3}$ próximo aos exaustores, em função do sistema de ventilação tipo túnel de pressão negativa, que faz o encaminhamento do ar no sentido longitudinal do aviário FIGURAS 5 e 6 (CARVALHO et al., 2012; SILVA et al., 2013). Os níveis de dióxido de carbono podem ser utilizados para a definição das taxas de ventilação necessárias em aviários de frango de corte (CALVET, 2011). 


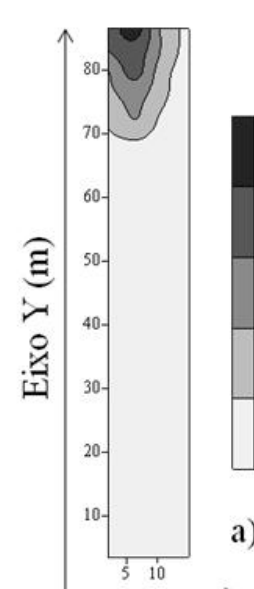

a)

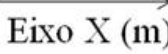

Distribuição da concentração de $\mathrm{CO}_{2}[\mathrm{ppm}]$
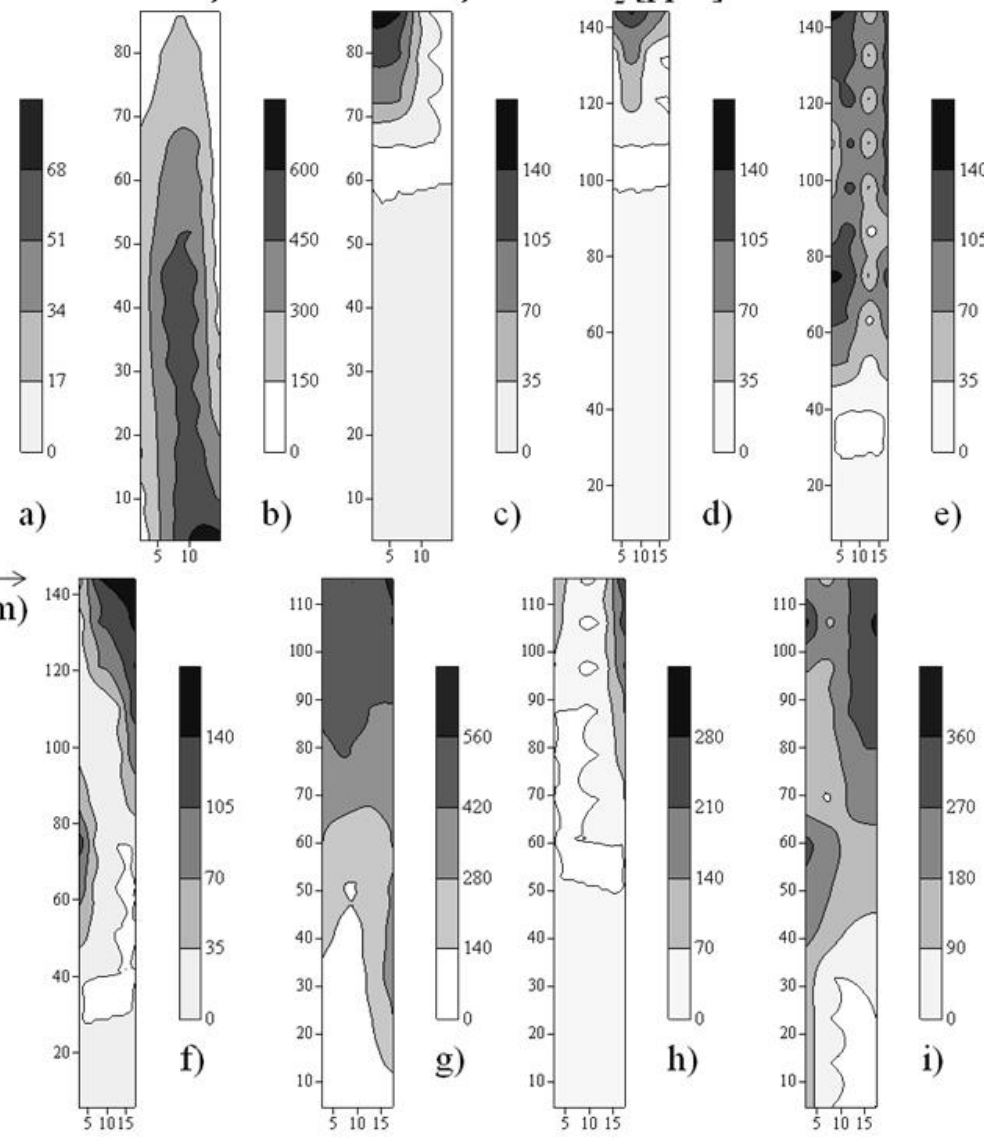

FIGURA 5. Mapas de krigagem para a concentração de dióxido de carbono no aviário BH, aos 28 (a), 35(b) e 42(c) dias de idade das aves, DH aos 28 (d), 35(e) e 42(f) dias de idade das aves, e SW aos 28 (g), 35(h) e 42(i) dias de idade das aves, no período do verão, às 14h. Kriging maps for $\mathrm{CO}_{2}$ concentration in $\mathrm{BH}$ in 28 (a), 35 (b) and 42 (c) days old of birds, DH in 28 (d), 35 (e) and 42 (f) days old of birds and SW in 28 (g), 35 (h) and 42 (i) days old of birds on summer and at 2 PM.

A concentração de $\mathrm{NH}_{3}$ esteve abaixo do limite máximo considerado ideal para todos os aviários, abaixo de 20 ppm (GLOBALGAP, 2012), embora os aviários SW, aos 42 dias de idade das aves, estivesse acima do limite máximo de $10 \mathrm{ppm}$ preconizado pelo manual de frango de corte da linhagem (COBB, 2013). Os mapas de distribuição da concentração de $\mathrm{NH}_{3}$ apresentaram níveis mais altos próximos aos exaustores, de forma análoga aos mapas de concentração de $\mathrm{CO}_{2}$ (FIGURA 6). 


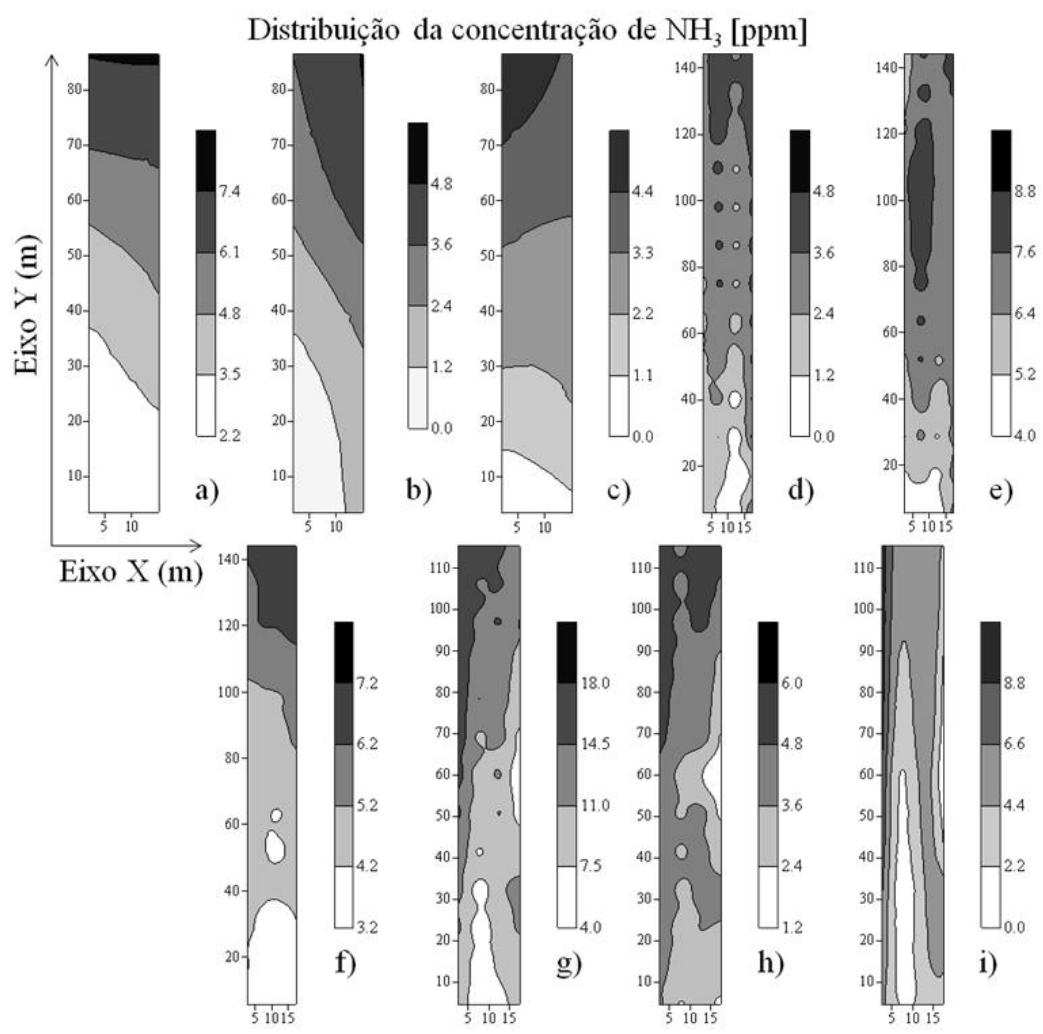

FIGURA 6. Mapas de krigagem para a concentração de amônia no aviário BH, aos 28 (a), 35(b) e 42(c) dias de idade das aves, DH aos 28 (d), 35(e) e 42(f) dias de idade das aves, e SW aos 28 (g), 35(h) e 42(i) dias de idade das aves, no período do verão às $14 \mathrm{~h} 00 \mathrm{~min}$. Kriging maps for $\mathrm{NH}_{3}$ concentration in $\mathrm{BH}$ in 28 (a), 35 (b) and 42 (c) days old of birds, DH in 28 (d), 35 (e) and 42 (f) days old of birds and SW in 28 (g), 35 (h) and 42 (i) days old of birds on summer and at 2 PM.

\section{CONCLUSÕES}

Por meio da análise geoestatística, observou-se que o sistema de ventilação utilizado nos diferentes aviários foi capaz de manter a qualidade do ar em relação à concentração de amônia e dióxido de carbono, embora não suficiente para a manutenção da ambiência em condições adequadas à criação das aves. A temperatura e a umidade relativa apresentaram-se além do adequado nos aviários estudados, com exceção do aviário $\mathrm{BH}$, que apresentou umidade aquém. A velocidade do ar esteve em níveis adequados para os aviários $\mathrm{BH}$ e $\mathrm{DH}$, e aquém para o $\mathrm{SW}$, remetendo que a presença ou a ausência do painel evaporativo influencia na condição-padrão de funcionamento do sistema de ventilação. O sistema de ventilação com sistema de resfriamento por painel evaporativo auxilia no resfriamento do ar no centro do aviário, enquanto nas paredes laterais ocorrem temperaturas acima do recomendado pela literatura.

\section{REFERÊNCIAS}

AMARAL, A.G.; YANAGI JUNIOR, T.; LIMA, R.R.; TEIXEIRA, V.H.; SCHIASSI, L. Effect of the production environment on sexed broilers reared in a commercial house. Arquivo Brasileiro de Medicina Veterinária e Zootecnia, Belo Horizonte, v.63, n.3, p.649-658, jun. 2011.

ARAUJO, R.B.; FARIA, D.E.; FARIA FILHO, D.E.; LIMA, C.G.; TREVISAN, R.B.; SOUZA, K.M.R.; SAKAMOTO, M.I.; SOUZA, V.N. Modelos de superfície de resposta para predição do desempenho de frangos e elaboração de análise econômica. Revista Brasileira de Saúde e Produção Animal, Salvador, v.12, n.3, p.770-783, 2011. 
BARBOSA FILHO, J.A.D.; VIEIRAI, F.M.C.; SILVA, I.J.O.; GARCIA, D.B.; SILVA, M.A.N.; FONSECA, B.H.F. Transporte de frangos: caracterização do microclima na carga durante o inverno. Revista Brasileira de Zootecnia, Viçosa, MG, v.38, n.12, dez. 2009.

BORGES, G.; MIRANDA, K.O.S.; RODRIGUES, V.C.; RISI, N. Uso da geoestatística para avaliar a captação automática dos níveis de pressão sonora em instalações de creche para suínos.

Engenharia Agrícola, Jaboticabal, v.30, n.3, p.377-385, 2010.

BOURNET, P.E.; BOULARD, T. Effect of ventilator configuration on the distributed climate of greenhouses: A review of experimental and CFD studies. Computers and Electronics in Agriculture, Amsterdam, n.74, p.195-217, 2010.

BUCKLIN, R.A.; JACOB, J.P.; MATHER, F.B.; LEARY, J.D.; NÄÄS, I.A. Tunnel ventilation of broiler houses. Gainesville: Institute of food and Agricultural Sciences, 2009. (FACTSHEET PS 46).

BUSTAMANTE, E.; GARCÍA-DIEGO, F.J.; CALVET, S.; ESTELLÉS, F.; BELTRÁN, P.; HOSPITALER, A.; TORRES, A.G. Exploring ventilation efficiency in poultry buildings: The validation of computational fluid dynamics (CFD) in a cross-mechanically ventilated broiler farm. Energies, Singapura, v.6, p.2605-2623, abril 2013.

CALVET, S.; ESTELLÉS, F.; CAMBRA-LÓPEZ, M.; TORRES, A.G.; VAN DEN WEGHE, H.F. The influence of broiler activity, growth rate, and litter on carbon dioxide balances for the determination of ventilation flow rates in broiler production. Poultry Science, Oxford, v.90, p.24492458, 2011.

CAMBARDELLA, C.A.; MOORMAN, T.B.; NOVAK, J.M. et al. Field scale variability of soil properties in Central Iowa soils. Soil Science Society of America Journal, Madison, v.58, n.5, p.1501-1511, 1994.

CARVALHO, T.M.R.; MOURA, D.J.; SOUZA, Z.M.; SOUZA, G.S.; BUENO, L.G.F. Litter and air quality in different broiler housing conditions. Pesquisa Agropecuária Brasileira, Brasília, v.46, n.4, p. 351-361, 2011.

CARVALHO, T.M.R., MOURA, D.J.; SOUZA, Z.M.; SOUZA, G.S.; BUENO, L.G.F.; LIMA, K.A.O. Use of geostatistics on broiler production for evaluation of different minimum ventilation systems during brooding phase. Revista Brasileira Zootecnia, Viçosa, MG, v.41, n.1, p.194-202, jan.2012.

CHOWDHURY, S.; THEMUDO, G.E.; SANDBERG, M.; ERSBØLL, A.K. Spatio-temporal patterns of Campylobacter colonization in Danish broilers. Epidemiology and Infection, Cambridge, v.141, n.5, p.997-1008, 2013.

COBB. Suplemento de crescimento e nutrição para frangos de corte. Massachusetts, 2013. 14 p. Disponível em: <http://www.cobb-vantress.com/docs/default-source/cobb-700-guides/cobb700broiler-performance-and-nutrition-supplement---portuguese.pdf?sfvrsn=0>. Acesso em: 15 jul. 2014.

CORKERY, G.; WARD, S.; KENNY, C.; HEMMINGWAY, P. Monitoring Environmental Parameters in Poultry Production Facilities. Computer Aided Process Engineering, Graz, v. 1, p.110, 2013. Disponível em < http://hdl.handle.net/10197/4257>. Acesso em: 24 nov. de 2013.

DOZIER, W.A.; PURSWELL, J.L.; BRANTON, S.L. Growth responses of male broilers subjected to high air velocity for either twelve or twenty-four hours from thirty-seven to fifty-one days of age. The journal of applied poultry research, Oxford, v.15, p.362-366, 2006.

GLOBALG.A.P. Integrated farm assurance / poultry. Controls Points and compliance criteria. Cologne, 2012. 54 p. Disponível em <www.globalgap.org>. Acesso em: 24 fev. de 2012. 
LITTLE, T.M.; HILLS, F.J. Agricultural experimentation: design and analysis. New York: John Wiley \& Sons, 1978. 368p.

McBRATNEY, A.B.; WEBSTER, R. How many observations are needed for regional estimation of soil properties? Soil Science, Philadelphia, v.135, n.3, p.177-183, 1983.

MEDEIROS, C.M.; BAÊTA, F.C.; OLIVEIRA, R.F.M.; TINÔCO, I.F.F.; ALBINO, L.F.T.;

CECON, P.R. Índice térmico ambiental de produtividade para frangos de corte. Revista Brasileira de Engenharia Agrícola e Ambiental, Campina Grande, v.9, n.4, p. 660-665, 2005.

MELUZZI, A.; SIRRI, F. Welfare of broiler chickens. Italy Journal of Animal Science, Pavia, v.8, n.1, p.161-173, mar. 2009.

MOSTAFA, E. et al. Computational fluid dynamics simulationof air temperature distribution inside broiler building fitted with duct ventilation system. Biosystems Engineering, Queensland, v.112, p.293-303, 2012.

NAZARENO, A.C.; PANDORFI, H.; ALMEIDA, G.L.P.; GIONGO, P.R.; PEDROSA, E.M.R.; GUISELINI, C. Avaliação do conforto térmico e desempenho de frangos de corte sob regime de criação diferenciado. Revista Brasileira de Engenharia Agrícola e Ambiental, Campina Grande, v.13, n.6, p.802-808, 2009.

NICHOLSON, F. A.; CHAMBERS, B. J.; WALKER, A. W. Ammonia Emissions from Broiler Litter and Laying Hen Manure Management Systems. Biosystems Engineering, Queensland, v.89, n.2, p.175-185, 2004.

PEREIRA, P.A.; YANAGI JUNIOR, T.; SILVA, J.P.; LIMA, R.R.; CAMPOS, A.T.; ABREU, L.H.P. Technical evaluation of artificial ligthing systems for broiler houses. Engenharia Agrícola, Jaboticabal, v.32, n.6, p.1011-1024, nov./dez. 2012.

ROCHA, H.P.; FURTADO, D.A.; NASCIMENTO, J.W.B.; SILVA, J.H.V. Índices bioclimáticos e produtivos em diferentes galpões avícolas no semiárido paraibano. Revista Brasileira de Engenharia Agrícola e Ambiental, Campina Grande, v.14, n.12, p.1330-1336, 2010.

SILVA, E.G.; SANTOS, A,C.; FERREIRA, C.L.S.; SOUSA, J.P.L.; ROCHA, J.M.L.; SILVEIRA JÚNIOR, O. Variabilidade espacial das características ambientais e peso de frangos de corte em galpão de ventilação negativa. Revista Brasileira de Saúde e Produção Animal, Salvador, v.14, n.1, 2013.

SAS INSTITUTE. Statistical analysis system for Windows: computer program manual. Cary, 1995. 705p.

SILVA, I. M.; PANDORFI, H.; ALMEIDA, G. L. P.; GUISELINI, C.; CALDAS, A. M.; JACOB, A. L. Análise espacial das condições térmicas do ambiente pré-ordenha de bovinos leiteiros sob regimes de climatização. Revista Brasileira de Engenharia Agrícola e Ambiental, Campina Grande, v.16, n.8, 2012.

TRANGMAR, B. B.; YOST, R. S.; UEHARA, G. Application of geostatistics to spatial studies of soil properties. Advances in Agronomy, Newark, v.38, n.1, p.45-93, 1985.

VIEIRA, S.R. Geoestatística em estudos de variabilidade espacial do solo. In: NOVAIS, R.F.; ALVARES, V.H.; SCHAEFER, C.E.G.R. Tópicos em ciência do solo. Viçosa: Sociedade Brasileira de Ciência do Solo, 2000. v.1, p.1-53.

WARRICK, A.W.; NIELSEN, D.R. Spatial variability of soil physical properties in the field. In: HILLEL, D. Applications of soil physics. New York: Academic,1980. p.319-344, 1980.

YANAGI JÚNIOR, T.; AMARA, A.G.; TEIXEIRA, V.H.; LIMA, R.R. Spatial characterization of thermoacoustic and luminance environment in commercial broiler chicken house. Engenharia Agrícola, Jaboticabal, v.31, n.1, p.1-12, jan./feb. 2011. 